\title{
Occipital nerve stimulator systems: Review of complications and surgical techniques
}

\author{
Steven Falowski \\ Thomas Jefferson University Hospital \\ Dajie Wang \\ Thomas Jefferson University Hospital \\ Arvind Sabesan \\ Thomas Jefferson University Hospital \\ Ashwini Sharan \\ Thomas Jefferson University Hospital
}

Follow this and additional works at: https://jdc.jefferson.edu/neurosurgeryfp

Part of the Medical Neurobiology Commons, and the Surgery Commons

Let us know how access to this document benefits you

\section{Recommended Citation}

Falowski, Steven; Wang, Dajie; Sabesan, Arvind; and Sharan, Ashwini, "Occipital nerve stimulator systems: Review of complications and surgical techniques" (2010). Department of

Neurosurgery Faculty Papers. Paper 6.

https://jdc.jefferson.edu/neurosurgeryfp/6

This Article is brought to you for free and open access by the Jefferson Digital Commons. The Jefferson Digital Commons is a service of Thomas Jefferson University's Center for Teaching and Learning (CTL). The Commons is a showcase for Jefferson books and journals, peer-reviewed scholarly publications, unique historical collections from the University archives, and teaching tools. The Jefferson Digital Commons allows researchers and interested readers anywhere in the world to learn about and keep up to date with Jefferson scholarship. This article has been accepted for inclusion in Department of Neurosurgery Faculty Papers by an authorized administrator of the Jefferson Digital Commons. For more information, please contact: JeffersonDigitalCommons@jefferson.edu. 


\title{
As submitted to:
}

Neuromodulation

And later published as:

"Occipital Nerve Stimulator Systems: Review of
Complications and Surgical Techniques"

\author{
Neuromodulation \\ Volume 13, Issue 2, April 2010, Pages 121-125 \\ DOI: $10.1111 / j .1525-1403.2009 .00261 . x$
}

\author{
Steven Falowski, Dajie Wang, Arvind Sabesan and Ashwini Sharan
}

\begin{abstract}
:
Stimulation of the occipital nerves is becoming more widely accepted in the treatment of occipital neuritis and migraine disorders. Presently, equipment available for spinal cord stimulation is adapted for insertion into the subcutaneous space over the occipital nerves. Many technical factors need to be reassessed to optimize the therapy. We performed a retrospective review of patients implanted from 2003-2007 at a single center. We aimed to analyze the rate of surgical complications related to implantation technique. A total of 28 patients were present for analysis. Patients were followed up to 60 months with a mean follow up of 21 months. There is a $32 \%$ revision rate for electrode migration or displacement, $3.6 \%$ removal rate for infection, and a $21 \%$ removal rate for lack of efficacy. Although not well studied secondary to small patient populations, this was consistent with a review of the literature which demonstrated a 10$60 \%$ revision rate. Other factors such as anchoring strategy, strain relief, and battery location were all considered in the analysis and will be presented. A major determination was that use of a second incision with an additional strain relief loop had only a $10 \%$ revision rate of the lead while those without this additional strain relief loop had a $62.5 \%$ revision rate. Many technical factors need to be addressed for optimization of occipital nerve stimulation.
\end{abstract}

\section{Introduction:}


The treatment of chronic neuropathic pain in the craniofacial region continues to be a challenge. Peripheral nerve stimulation has been attempted to treat craniofacial pain for these types of patients. Although the use of peripheral nerve stimulators is a not a novel concept, its use in the craniofacial region is relatively recent.

Multiple studies have shown the effective treatment of occipital neuralgia with stimulation of the occipital nerve $(\mathbf{6 , 7 , 8})$. Slavin et al. have described benefits in $70 \%$ of the patients treated for occipital neuralgia.(6) Similar results were reported by Melvin et al, in which $91 \%$ of the patients treated for $\mathrm{C} 2$ mediated occipital headaches reported reduction in medication use as well as $64 \%$ of the patients experienced reduction in the number of headaches (7). Other studies have also shown the efficacy of occipital nerve stimulation for the treatment of migraine headaches $(\mathbf{2 , 9 , 1 0 )}$. Schwedt et al. reported that 9 out of 15 patients $(60 \%)$ treated for various chronic headache syndromes experienced a $30 \%$ reduction in headache severity and frequency (2). In another study, $85 \%$ of patients with transformed migraine showed a greater than $50 \%$ reduction in frequency or severity of the headache after stimulation (9). Further studies are being carried out to further investigate the effectiveness of occipital stimulation for other headache disorders (10).

Equipment designed for spinal cord stimulation equipment is used for implantation into the occipital region over the distal branches of occipital nerves to induce an anti-nocioceptive effect. Occipital nerve stimulation as such is susceptible to the complications that have been described with the use of spinal cord stimulators. These complications include, lead migration, infection, pain, as well as device failure (1). The most common of these complications is lead migration and often requires surgical intervention $\mathbf{( 1 , 1 3 , 1 4 ) . ~ I n ~ 1 9 9 3 , ~ i n ~ a ~ s t u d y ~ b y ~ N o r t h ~ e t ~ a l . ~ w h i c h ~ d e s c r i b e d ~ a ~} 20$ year experience, lead migration was found in $22 \%$ of patients although the percentage of patients that needed surgical intervention was not disclosed. (12). In another review of the literature by Ubbink et al., $14.8 \%$ of patients required surgical intervention due to lead migration or fracture (13).

With occipital nerve stimulation, there has also been a significant lead migration requiring subsequent surgical intervention. Slavin et al. have reported $10 \%$ patients experiencing lead migration which required surgical intervention (6). Similarly, Melvin et al have reported $9 \%$ lead migration (7). Popeney et al. described a slightly higher rate at $3 / 25$ patients (12\%) having spontaneous lead migration which required surgical intervention. However, in a study of 15 patients done by TJ Schwedt et al, there was a $33 \%$ lead migration 6 months post implantation, 60\% migration after 2 years, and 100\% migration after 3 years.(2). Due to a lack of studies with large sample size it is hard to quantify the percentage of patients that will experience lead migration and subsequent surgical intervention.

Another serious complication is infection at the surgical site. The rate of infection has ranged from $2 \%-10 \%$ in patients who received implantable pain therapy devices such as spinal cord stimulators $(\mathbf{3 , 4 , 5})$. Finally other complications can occur with the device such as electrical failure, loss of charge, or programming issues.

The purpose of this study is not to discuss the efficacy of occipital nerve stimulators, but is to analyze the rate of surgical complications related to implantation technique specific to occipital nerve stimulation and compare this to complication rates observed in the literature for peripheral nerve stimulation. 


\section{Methods:}

After attaining institutional review board approval, a retrospectively chart review was performed. All patients who had undergone occipital nerve stimulation from 2003- 2007 were included in the review. 28 patients met criteria for inclusion within the study. Ages ranged from 22-63 years with 25 female and 3 male patients. Average age of patients was 41 years. Table 1 further details the patient demographics. Follow up ranged from 6-60 months with a mean follow-up period of 21 months. The analysis was performed based on surgical details, complications, and reports from repeated operations. Patient satisfaction as well as efficacy of the stimulation was not analyzed.

\section{Implantation}

Device: The peripheral nerve system implanted consisted of an implantable pulse generator, as well as a percutaneous lead with use of extensions. Equipment form three manufacturers were used. These included Medtronic, Advanced Neuromodulation Systems(ANS) and ABS. All subjects initially received 2 conventionally placeable percutaneously wire leads with either 4 contacts or 8 contacts placed through a midline incision between the base of the occiput and C2. The IPG utilized was either a 8 or 16 channel programmable device. Anchors provided by the specific manufacturer were used.

\section{Trial:}

All patients had received a pre- implantation trial. However, these trials were not all standardized. Twenty patients received an outpatient trial over three to five days prior to implantation. Eight patients had only an on table trial. All patients were implanted within 4 weeks of their trial. Those implanted with ANS(16) or ABS(4) received a trial prior to implantation while those with a Medtronic implant(8) had an on- table trial. All patients reported greater than 50 percent of headache reduction during these trials.

\section{Surgical Implantation:}

Patients were implanted either under general anesthesia or under intravenous sedation with local anesthesia. The patients were placed in a prone position if under general anesthesia or positioned in the lateral position if done under IV sedation.

A midline $2 \mathrm{~cm}$ incision was made at the level of $\mathrm{C} 1$ and an approximate $2 \mathrm{~cm}$ subcutaneous pocket was created above and visualizing the occipital cervical fascia. A Touhy needle was advanced laterally on the skull from the midline incision in a trajectory over the occipital nerve. If the patient were under general anesthesia, the trajectory was determined by insonating the occipital artery aiming the Touhy needle across that region $(16,17)$. If the patient were under sedation, awake testing was performed to test for maximal stimulation of the occipital region during intra-operative testing. Multiple repositions were potentially performed to achieve this. The needle was used to place the lead. The lead was then secured via an anchor placed proximal to the electrode. The anchor was secured to the fascia and to the electrode with two neurolon sutures. In every case, a strain relief loop was tucked into the subcutaneous fascia after creating an area with less tension. 
The IPG was then placed via an incision in the buttocks, chest, or abdomen and the location was determined based on the patient's preference. Leads were tunneled to an extension when needed. Later in the learning curve, a paraspinal incision was added and an additional subcutaneous pocket was created in the region around C7- T1 where an additional strain relief loop was placed and secured with a stitch. There was then further tunneling to the IPG. Incisions were then closed in two layers. The patient were all taken to the recovery room and subsequently discharged home on the same day.

\section{Results:}

Twenty-eight patients were included for evaluation. Follow up was 2-60 months with a mean of 21 months. Follow up included office visits at 1 week, 1 month, 1 year, and 2 years with X-rays for evaluation as well as any additional needed office visits. Age of patients ranged from 22-63 years with 25 female and 3 male patients. Average age of patients was 41 yo.

The most common adverse event was lead migration (Table1). This was observed in 7 of the 28 patients and they were all females. In fact, there were a total of 13 revisions surgeries in those 7 patients. This was observed over the continuum of the 60 month follow up period demonstrating it to be a phenomenon seen well after the acute period of implantation. There was a 26 week average time to revision with 54\% requiring revision within 8 weeks, 39\% within 4 weeks and $31 \%$ within 2 weeks. All 13 lead migration required surgical revision. It is of importance to note that only 3 of the observed lead migrations ( $23 \%$ of the migrations) were secondary to documented traumas which lead to the displacement of the leads. One of these was a facial trauma which caused hyperextension of the neck and a broken lead. Another was a fall while standing into a door causing flexion- rotation of the neck which caused displacement of the lead. While the third was observed after a motor vehicle accident. In all patients treated for migration the effectiveness of the stimulator was restored after revision and no residual side-effects were noted.

Over time the surgical technique was modified to include an additional strain relief loop placed via a second incision in the cervical-thoracic paraspinal region. Use of a second incision with an additional strain relief loop had a $10 \%$ revision rate of the lead while those without this additional strain relief loop had a $62.5 \%$ revision rate. The two revisions (10\%) noted with a second strain relief loop were also observed only after documented trauma. The revision rate with those patients undergoing a second strain relief loop excluding trauma would be $0 \%$ in the current review.

Initial electrode revision for migration was noted in 7 patients or $25 \%$ of total patients. Five of these seven patients would undergo revision surgery again for electrode revision (71\%). Repeat revisions were only noted in those patients undergoing placement without a second strain relief loop. Of note the two others not requiring a repeat revision were 
patients who had initially been placed with a second strain relief loop but sustained a trauma.

Other adverse events included infection. Five of the 28 patients (17.9\%) developed signs of an infection. Four of the 5 patients $(14.3 \%)$ were successfully treated with PO antibiotics with follow up in the office Two of the four patients treated with PO antibiotics were at the IPG site (one abdomen, one buttock) while another was at the cervical incision and the last was at the second incision lateral to C7-T1. One patient (3.6\%) required removal of the electrode and IPG with replacement in 2 months after a 6 week course of IV antibiotics. This patient had erosion of the wires through the skin of her back.

In 6 patients $(21 \%)$ the stimulator was removed for lack of efficacy. 5 of these 6 patients felt that the stimulation was not effective while the sixth did not like the sensation/parasthesias accompanying the stimulation. The degree of efficacy and patient satisfaction with the stimulation is not evaluated in this study.

Two patients $(7.1 \%)$ were noted to have persistent pain at the incisional sites. One patient described pain in the cervical region which did not require surgical correction. The anchors appeared to become palpable through the skin. The other patient (3.6\%) was noted to have pain at the battery site in the buttock. This patient even underwent repositioning of the battery to the abdomen but still continues to have pain at the battery site despite surgical re-exploration of that site. Incidentally, he is still continuing to use his neural stimulation system to date.

Four patients were noted to have pain at their battery site. Three of these were in the buttock from which there were 21 implants (14.2\%) while one was in the chest for which there were 5 implants $(20 \%)$. Two of the three batteries placed in the buttock that had pain required surgical revision. There were a total of 4 abdominal battery placements, of which two were after revision from initial buttock placement. None of these patients reported pain at their battery site.

Battery revision for migration was also noted. There was one migration in the chest $(20 \%$ of chest implants) as well as one migration in the buttock (4.8\% of buttock implants). Therefore there is a $7.1 \%$ battery migration rate noted across all patients and battery placements. Migration was not noted in the four abdominal implants.

Of the remaining surgeries performed there was an IPG change in 4 patients (14\%) for end of life of the battery. There was also a revision of the IPG secondary to malfunction of the battery in 1 patient (3.6\%). This was observed after a fall in which the patient landed on the battery. There was also a revision of the lead secondary to malfunction in 1 patient $(3.6 \%)$.

In the 28 patients there were a total of 59 surgeries performed including those in reference to the battery, while there was 41 surgeries total in reference to the leads. Each patient underwent between 1 and 5 surgeries in the follow up period of 60 months. 
Analyzing this data demonstrates that on average there was 2.1 surgeries per patient in a 60 month follow up period.

\section{Discussion:}

Occipital nerve stimulation is now being investigated for the treatment of chronic headache syndromes $(2,7,9,10)$. Additionally, multiple previous studies have shown the effective treatment of occipital neuralgia with peripheral stimulation of the occipital nerve $(\mathbf{6 , 7 , 8})$. The effectiveness of therapy will be dependent on patient population and disease entity being treated and hopefully borne out in prospective clinical trials. However, the effectiveness of the therapy is highly also influenced by its surgical complications.

Occipital nerve stimulation can share some of the various complications that arise with the use of spinal cord stimulators. These complications include lead migration, infection, as well as device failure (1). The most common of these complications is lead migration which has also been reported with spinal cord stimulators and this often requires surgical intervention $(\mathbf{1}, \mathbf{1 3}, \mathbf{1 4})$. The revision rate for peripheral nerve stimulation appears to be

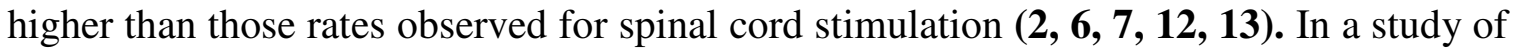
15 patients with occipital nerve stimulation done by Schwedt et al, there was a $33 \%$ lead migration 6 months post implantation, $60 \%$ migration after 2 years, and 100\% migration after 3 years.(2). Schwedt et al commented that surgical revision may be more common after an adequate length of follow up. However, it is expected that most patients will have lead migration requiring revision within 1 year of implantation. Oh et al found a $70 \%$ revision rate for migration in occipital nerve stimulators within the first 6 weeks of implantation (15). In this paper they believe that the use of percutaneous cylindrical electrodes led to electrode migration secondary to anchor dislodgement from recurring skull base spasm. Other papers have also commented on higher rates of migration with the use of cylindrical electrodes in the high cervical region $(\mathbf{9}, \mathbf{1 4})$. It was therefore felt that use of a paddle style electrode was less likely to migrate secondary to the ability for easier and more effective suturing or anchoring to the fascia or subcutaneous tissue (15).

In the current series, the most common adverse event was lead migration (Table1). These were seen in 7 of the 28 patients followed for a total of 13 revisions within 41 surgeries (32\%). This was observed over the continuum of the 60 month follow up period confirming that lead migration may be seen in most patients in the acute period but may also occur over extended periods of follow up. It is of importance to note that 3 of the observed lead migrations ( $23 \%$ of the migrations) were secondary to documented traumas which lead to the displacement of the leads.

Methods of implantation and anchoring differ among surgeons. There is an evolution over time that leads to changes in technique for anchoring as well as positioning of the electrode. Important distinctions can be made on the choice of suturing to the subcutaneous tissue versus the cervical fascia. Placement and methods of stress relief of the leads can also effect the amount of lead migration sustained. Lastly the location and placement of the IPG can change degree of strain on the electrode when considering angle and direction of tunneling as well as distance from the electrode site. 
In this series, the use of an additional strain relief loop through a separate incision dramatically reduced the lead migration and revision rate. Patients with an additional strain relief loop had only a $10 \%$ revision rate of the lead while those without this additional strain relief loop had a $62.5 \%$ revision rate. Furthermore, the two revisions $(10 \%)$ noted with a second strain relief loop were also observed after a documented trauma leading to the assumption that without associated trauma the revision rate would be much lower.

It has been observed that there is less migration rates when using a midline incision as oppose to a lateral incision (7). A midline incision dictates the use of two electrodes if there is a need for bilateral stimulation. A single electrode can be used to span both occipital nerves if a lateral incision is utilized. However it is felt that use of the midline incision will allow more effective placement, ease of placement, and a greater ability to anchor the electrode in a position that allows minimal migration, if any. Use of two electrodes will also allow a greater area of coverage that cannot be obtained via a single electrode that spans both occipital nerves.

Some patients were noted to have pain at their battery site. The majority of these were noted with placement in the buttock. Two of the three batteries placed in the buttock that had pain required surgical revision ( $7.1 \%$ total or $9.5 \%$ of buttock). There were a total of 4 abdominal battery placements, of which two were after revision from initial buttock placement. None of these patients reported pain at their battery site. Although a small number this suggests that patients tolerate placement of the IPG in the abdomen over the buttock. It did not appear in our study to affect migration or revision rates. The drawback to abdominal placement of the IPG is that it necessitates placement in the lateral position in the operating room as oppose to prone when undergoing buttock placement.

Each patient underwent between 1 and 5 surgeries in the follow up period of 60 months. Analyzing this data demonstrates that on average there was 2.1 surgeries per patient in a 60 month follow up period. One potential area of improvement is the type of equipment. PNS systems are usually modified forms of spinal cord stimulation equipment. Although there is interchangeability among the two it has become more evident that use of this equipment has different rates of migration between use as a PNS and a SCS. It is therefore feasible that with new equipment and technology we can limit the lead migration rate by designing systems that are more specific to PNS. More specifically stimulation in the cervical region leads to cervical tension as well as skull base spasm that is not seen in other sites of PNS and this has to be taken into account when considering causes of anchor dislodgement.

Another serious complication is infection at the surgical site. Historically, the rate of infection has ranged from $2 \%-10 \%$ in patients who received implantable pain therapy devices such as spinal cord stimulators $(\mathbf{3}, \mathbf{4 , 5})$. Our data showed a $3.6 \%$ removal rate for infection and $17.9 \%$ overall rate of infection. 4 of the 5 patients $(14.3 \%)$ were successfully treated with oral antibiotics with follow up in the office. Two of the four patients treated with oral antibiotics were at the IPG site while another was at the cervical 
incision and the last was at the second incision lateral to C7-T1. There did not seem to be a common factor, such as location of battery or placement of leads that lead to infection. This is consistent with published data and seems to be interchangeable among SCS and PNS.

\section{Conclusion:}

The rate of surgical revision with occipital nerve stimulators remains high. This may be largely a factor of the differences in surgical techniques. A paddle style electrode implanted via a midline incision may be effective in lowering this rate. Additionally, better anchoring to the cervical fascia and the use of an second strain relief loop at the cervical-thoracic junction may overcome the cervical tension imposed on the electrodes. A consensus statement among implanting physicians critically analyzing the factors in surgical technique to minimize complications is due. Overtime if there is not a change in this rate of revision it will be interesting to evaluate if the effectiveness of occipital nerve stimulation as a treatment modality for various syndromes out weights the multiple surgeries required to maintain its sustainability. Finally, hardware related pain requiring re-operation will also need to be addressed by the manufacturers. 
Table 1a: Incidence of Adverse Events

Adverse Event
Lead Migration
Lead Migration secondary to
Trauma
Battery Migration
Infection-PO antibiotics
Infection-IV antibiotics
Lack Of Efficacy
Malfunction(Lead)
Malfunction(Battery)
EOL battery

Number of Patients Surgical Revision

Rate of 7 patients/13 revisions Yes

Revision

3 Yes

$32 \%$

$23 \%$ of

migrations

2 Yes

$7.1 \%$

4 No

$14.3 \%$

1 Yes

$3.6 \%$

6 Yes

$21 \%$

1 Yes

$3.6 \%$

1 Yes

$3.6 \%$

4 Yes

$14 \%$ 
Table 1b: Breakdown of Lead Migrations

\begin{tabular}{|c|c|c|c|c|}
\hline & $\begin{array}{l}\text { Number of } \\
\text { Patients(First Revision) }\end{array}$ & Percentage & $\begin{array}{l}\text { Number of Patients } \\
\text { Requiring Repeat } \\
\text { Revisions after First }\end{array}$ & Percentage \\
\hline Total Patients & 28 & $100 \%$ & 5 & $17.9 \%$ \\
\hline Total Lead & & & & \\
\hline $\begin{array}{l}\text { Revisions } \\
\text { Patients with } \\
\text { Strain Relief }\end{array}$ & 7 & $25 \%$ & 5 patients (6 revisions) & $71 \%$ \\
\hline $\begin{array}{l}\text { Loop } \\
\text { Strain relief loop }\end{array}$ & 20 & $71.5 \%$ & 0 & $0 \%$ \\
\hline $\begin{array}{l}\text { patients with lead } \\
\text { migration }\end{array}$ & $\begin{array}{r}2-\text { (Both included } \\
\text { trauma) }\end{array}$ & $\begin{array}{l}10 \%(7.1 \% \text { of } \\
\text { total patients })\end{array}$ & 0 & $0 \%$ \\
\hline $\begin{array}{l}\text { Patients without } \\
\text { Strain Relief }\end{array}$ & & & & \\
\hline $\begin{array}{l}\text { Loop } \\
\text { Without strain } \\
\text { relief loop } \\
\text { patients with lead } \\
\text { miaration }\end{array}$ & 8 & $\begin{array}{r}28.5 \% \\
62.5 \%(17.9 \% \\
\text { of total } \\
\text { patients })\end{array}$ & 6 revisions & $71 \%$ \\
\hline
\end{tabular}


Table 1c: Other Events

Buttock IPG Placement

Number

Chest IPG Placement

Abdomen IPG

Placement

2 initial $/ 4$ total

$75 \%$

$17.9 \%$

Pain at IPG(Total)

4

$7.1 \%$

Pain at IPG(Buttock)

3

$14.3 \%$

Pain at IPG(Chest)

1

$19.0 \%$

Pain at IPG(Abdomen)

4

$20.0 \%$

Battery Revision for

Pain(Total)

Battery Revision for

Pain(Buttock)

$7.1 \%$ of total

$9.5 \%$ of Buttock

2

placement

Pain at Electrode

1

$3.6 \%$

Electrode Revision For

Pain(at buttock incision)

1

$3.6 \%$ 
Table 2: Demographics

Total Number of Patients

Year of Implant

Gender

Mean Age(range)

Average Follow up(range)

Average Number of

Surgeries(total)
28, ANS(16) Medtronic(8) ABS(4)

2003- 2007

25 Female, 3 Male

41 years (22-63years)

21 months (6-60 months)

$2.1(59)$ 


\section{References:}

1) Trentman TL, Zimmerman RS. Occipital nerve stimulation: technical and surgical aspects of implantation. Headache. 2008 Feb; 48(2):319-27

2) Schwedt TJ, Dodick DW, Hentz J, Trentman TL, Zimmerman RS. Occipital nerve stimulation for chronic headache--long-term safety and efficacy. Cephalalgia. $2007 \mathrm{Feb} ; 27(2): 153-7$.

3) Ubbink DT, Vermeulen H, Spincemaille GH, et al. Systematic review and metaanalysis of controlled trials assessing spinal cord stimulation for inoperable critical leg ischemia. Br J Surg. 2004;91:948-955.

4) Spincemaille GH, Klomp HM, Steyerberg EW, et al. Technical data and complications of spinal cord stimulation: data from a randomized trial on critical limb ischemia. Stereotact Funct Neurosurg. 2000;74:63-72.

5) Burgher AH, Barnett CF, Obray JB, Mauck WD. Introduction of infection control measures to reduce infection associated with implantable pain therapy devices. Pain Pract. 2007 Sep;7(3):279-84

6) Slavin, Konstantin. Peripheral Neurostimulation for treatment of intractable occipital neuralgia. Neruosurgery 58:112-119 2006

7) Melvin, Euguene. Using Peripheral stimulation to reduce the pain of $c 2$ mediated occipital headaches: A preliminary report. Pain Physician 10:453-460 2007

8) Kapural, Leonardo. Occipital nerve electrical stimulation via the mideline approach and subcutaneous surgical leads for treatment of sever occipital neuralgia. A pilot Study. Anesth Analg 2005;101:171-174

9) Popeney CA, Aló KM, Peripheral neurostimulation for the treatment of chronic, disabling transformed migraine, Headache, 2003;43(4):369-75.

10) Webster, Lynn. Occipital Nerve Stimulation for Migraine. US Neurological Disease 2007 Issue 1 p33

11) Slavin, Konstantin. Trigeminal and occipital peripheral nerve stimuation for craniofacial apin; a single institution experience and review of the literature. Neurosurg Focus 21 (6): E6, 2006

12) North RB, Kidd DH, Zahurak M, James CS, Long DM. Spinal cord stimulation for chronic, intractable pain: Experience over two decades. Neurosurgery. 1993;32:384-394

13) Ubbink, D. Systematic review and meta-analysis of controlled trials assessing spinal cord stimulation for inoperable critical leg ischaemia. British Journal of surgery 2004; 91: 948-955

14) Weiner RL, Reed KL. Peripheral neurostimulation for the control of intractable occipital neuralgia. Neuromodulation 1999; 2:369-375. 
15) Oh MY, Ortega J, Bellotte JB, Whiting DM, Alo K. Peripheral nerve stimulation for the treatment of occipital neuralgia and transformed migraine using a C1-2-3 subcutaneous paddle style electrode: a technical report. Neuromodulation 2004; 7:103-12.

16) Shimizu, Satoru M.D.; Oka, Hidehiro M.D.; Osawa, Shigeyuki M.D.; Fukushima, Yutaka M.D.; Utsuki, Satoshi M.D.; Tanaka, Ryusui M.D.; Fujii, Kiyotaka M.D. Can Proximity of the Occipital Artery to the Greater Occipital Nerve Act as a Cause of Idiopathic Greater Occipital Neuralgia? An Anatomical and Histological Evaluation of the Artery-Nerve Relationship. Plastic and Reconstructive Surgery. Volume 119(7), June 2007, pp 2029-2034.

17) Jundt, JW. Mock, D. Temporal arteritis with normal erythrocyte sedimentation rates presenting as occipital neuralgia. Arthritis Rheum. 1991 Feb;34(2):217-9. 